\title{
Grayscale Medical Image Segmentation Method Based on 2D\&3D Object Detection with Deep Learning
}

\section{Yunfei Ge}

Tongji University

Qing Zhang

Tongji University

Yuantao Sun ( $\square$ sun1979@sina.com )

Tongji University

\section{Yidong Shen}

The First people's Hospital of Yancheng

\section{Xijiong Wang}

Shanghai Bojin Electric Instrument \& Device Co., Ltd

\section{Research Article}

Keywords: Grayscale medical image, Image segmentation, Deep learning, Object detection, Point cloud

Posted Date: October 29th, 2021

DOI: https://doi.org/10.21203/rs.3.rs-1018292/v1

License: (9) This work is licensed under a Creative Commons Attribution 4.0 International License. Read Full License

Version of Record: A version of this preprint was published at BMC Medical Imaging on February 27th, 2022. See the published version at https://doi.org/10.1186/s12880-022-00760-2. 


\title{
Grayscale Medical Image Segmentation Method Based on 2D\&3D Object Detection with Deep Learning
}

\author{
Yunfei Ge ${ }^{1}$, Qing Zhang ${ }^{1}$, Yuantao Sun ${ }^{1 *}$, Yidong Shen ${ }^{2}$, Xijiong Wang ${ }^{3}$ \\ 1 School of Mechanical Engineering, Tongji University, Shanghai, China \\ 2 Department of Orthopaedics, The First people's Hospital of Yancheng, Yancheng, China \\ 3 Shanghai Bojin Electric Instrument \& Device Co., Ltd, Shanghai, China
}

\begin{abstract}
Background: Grayscale medical image segmentation is the key step in clinical computer-aided diagnosis. Modeldriven and data-driven image segmentation methods are widely used for their less computational complexity and more accurate feature extraction. However, model-driven methods like thresholding usually suffer from wrong segmentation and noises regions because different grayscale images have distinct intensity distribution property thus pre-processing is always demanded. While data-driven methods with deep learning like encoder-decoder networks always are always accompanied by complex architectures which require amounts of training data.

Methods: Combining thresholding method and deep learning, this paper presents a novel method by using 2D\&3D object detection technologies. First, interest regions contain segmented object are determined with fine-tuning 2D object detection network. Then, pixels in cropped images are turned as point cloud according to their positions and grayscale values. Finally, 3D object detection network is applied to obtain bounding boxes with target points and boxes' bottoms and tops represent thresholding values for segmentation. After projecting to 2D images, these target points could composite the segmented object.
\end{abstract}

Results: Three groups of grayscale medical images are used to evaluate the proposed image segmentation method. We obtain the IoU (DSC) scores of $0.92(0.96), 0.88(0.94)$ and 0.94 (0.94) for segmentation accuracy on different datasets respectively. Also, compared with five state of the arts and clinically performed well models, our method achieves higher scores and better performance.

Conclusions: The prominent segmentation results demonstrate that the built method based on 2D\&3D object detection with deep learning is workable and promising for segmentation task of grayscale medical images.

Keywords: Grayscale medical image, Image segmentation, Deep learning, Object detection, Point cloud.

*Corresponding Author Yuantao Sun, E-mail: sun1979@ sina.com

\section{Background}

Medical imaging plays the key role in diagnosis or disease treatment by revealing internal structures with technologies mainly of computer tomography (CT), magnetic resonance imaging (MRI), ultrasound, and especially X ray radiography [1]. Due to different absorption capability of various organs or tissues for radiations, waves, and etc., pixels belong to various object in grayscale medical images have diverse grayscale values usually from 0-255 [2] and meanwhile values of pixels of the same object always gather within a range. 
38 Medical image segmentation has been widely applied to make images clearer with anatomical or

39 pathological structures changes [3], such as bone segmentation [4], lung segmentation [5,6], heart

40 fat segmentation [7] and liver or liver-tumor segmentation [8,9], etc. They could be considered to

41 divide origin images into several sub regions for picking up some crucial objects and extracting

42 interesting features which improve the computer aided diagnostic efficiency. There has raised

43 enormous approaches and they could be classified into two categories: model-driven techniques

44 and data-driven techniques. [5,10]

45 Many model-driven methods for medical image segmentation, including thresholding, clustering,

46 and region growing, were presented in particular before the widespread application of deep

47 learning. [10] Thresholding was one of the most common used method in practice due to its

48 efficiency. [11] The basic working of thresholding was to determine specific threshold values and

49 each pixel in the image could be classified as the foreground or background depending on the

50 comparison between their intensity values and threshold values. [12-14] Traditional thresholding

51 methods always relied on single models for universal segmentation tasks which could lead to

52 incorrect results. Also, segmentation objects often occupied only parts of whole images and pixels

53 of different objects may share same intensity values, so noises could appear if image segmentation

54 was applied overall.

55 With the era of big data coming, emerging data-driven technologies with deep learning have 56 remarkably demonstrated in variety medical image segmentation task. Supervised learning 57 methods and especially some CNN (Convolutional neural network) based encoder-decoder 58 structures such as FCN (Fully convolutional networks) [15], U-Net [16], DeepLab [17] has

59 practically proved [5]. Compared with traditional methods, deep learning could help analyze 60 medical images more effectively and extract more detailed features. 
61 Although these end-to-end structures was pragmatic for medical images semantic segmentation,

62 the segmentation accuracy always relied on a large amount of training dataset. But medical image

63 annotation could be time-consuming and quite expensive, thus transfer learning was used to solve

64 the problem of limited labeled data and pre-trained networks on natural images as ImageNet [18]

65 were often adopted for image segmentation. $[19,20]$ However, considering these datasets were

66 mainly designed to train models for object detection or classification, they may be more suitable

67 to pretrain networks for object detection. This inspired us to segment images with object detection.

68 We find that grayscale images could be segmented according to the comparison of thresholding

69 values with values of pixels in images and these pixels could be turned into 3D point cloud

70 according to their positions and grayscale values. Thus, by applying 3D object detection in the

71 point cloud, we could achieve groups of points within $3 \mathrm{D}$ bounding boxes. The top and the bottom

72 of boxes represent the thresholding values for segmentation and after mapping these points into

73 2D images, corresponding pixels could compose segmented results. Besides, 2D object detection

74 could determinate regions of interest (ROI) in grayscale medical images to reduce noises.

75 Therefore, according to above strategy, we propose the grayscale medical image segmentation

76 method based on 2D\&3D object detection.

77 The remainder of this paper is organized as following: Section 2 introduces the applied medical

78 Image datasets and describes details of proposed technologies, while in section 3 the obtained

79 results are displayed and the discussion is provided. Finally, section 4 presents the conclusions as

80 well as future work suggestions. 


\section{Methods}

82

83 Considering roles of grayscale images in medical field, two typical sets of available datasets are

84 prepared including musculoskeletal radiographs, and chest radiographs. Musculoskeletal

85 radiographs dataset (MURA (Musculoskeletal radiographs) \& LERA (Lower extremity

86 radiographs)) contains bone $\mathrm{X}$ ray images of upper and lower extremity. [21,22] Chest radiographs

87 dataset CheXpert (Chest radiography) has chest $\mathrm{X}$ ray images. [23,24]

88 The proposed grayscale medical image segmentation method is based on the supervised artificial

89 intelligence techniques, and labels are performed manually in two types medical images for model

90 training. Fig.1. shows origin images, and their respective Ground Truth (GT) images in different

91 datasets.

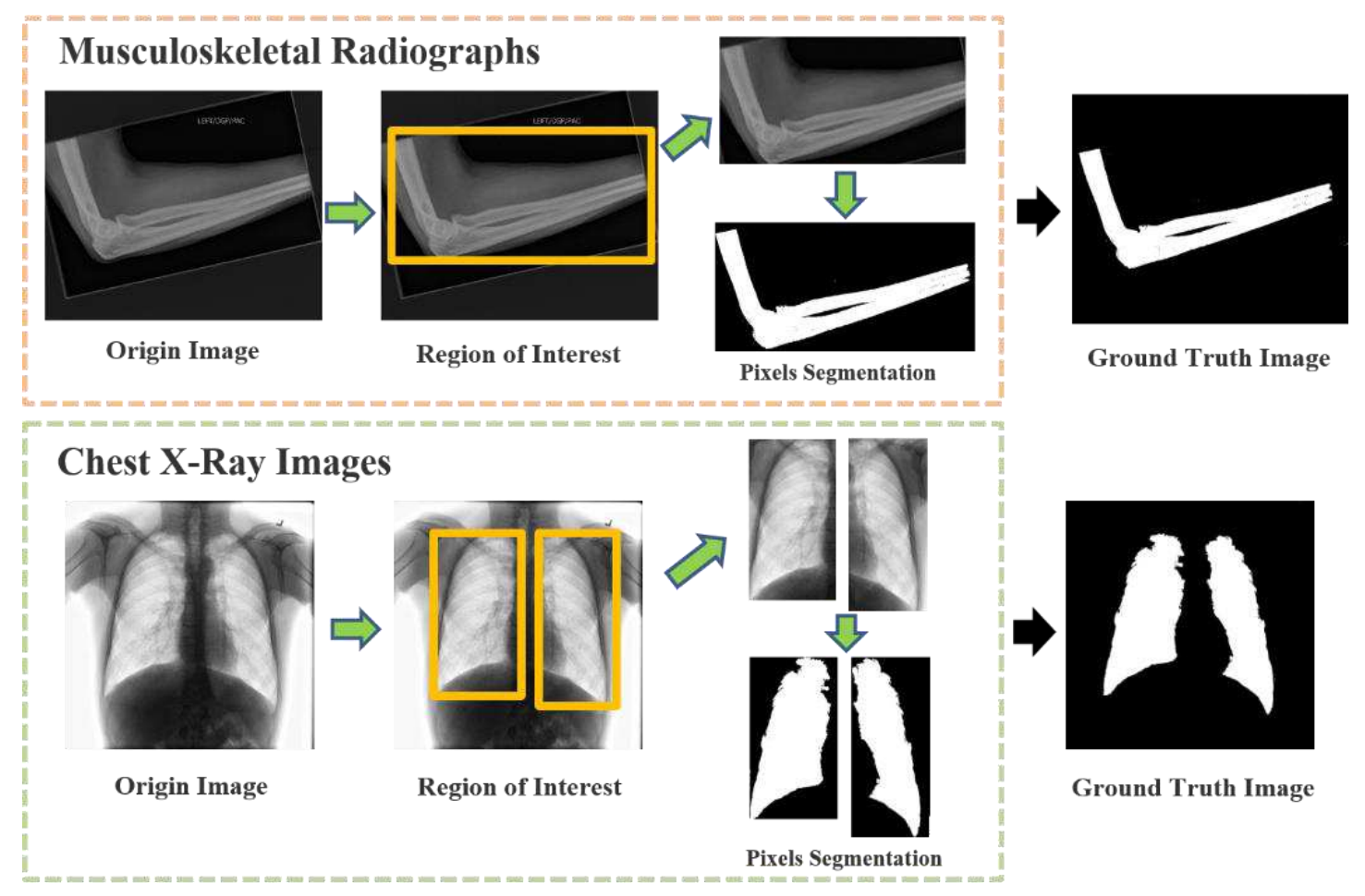


95 The proposed image segmentation method maps each pixel in the medical grayscale image to 3D

96 coordinates as the pixel-features point cloud, according to their positions and gray values. By

97 acquisition of foreground points and their corresponding bounding box using 3D object detection

98 method, we could achieve threshold values and the segmentation result of the corresponding

99 grayscale image. The whole pipeline and the implementation flow of this method are shown in

100 Fig.2 and Fig.3 respectively. Given a grayscale medical image, after (1) obtaining interest regions

101 of associated segmentation objects in the image, (2) generating 3D bounding box proposals in

102 point cloud and (3) the regression of their locations and scales, the refined boxes could be achieved.

103 The projection of points in refined bounding box into the $2 \mathrm{D}$ image is the segmentation result.

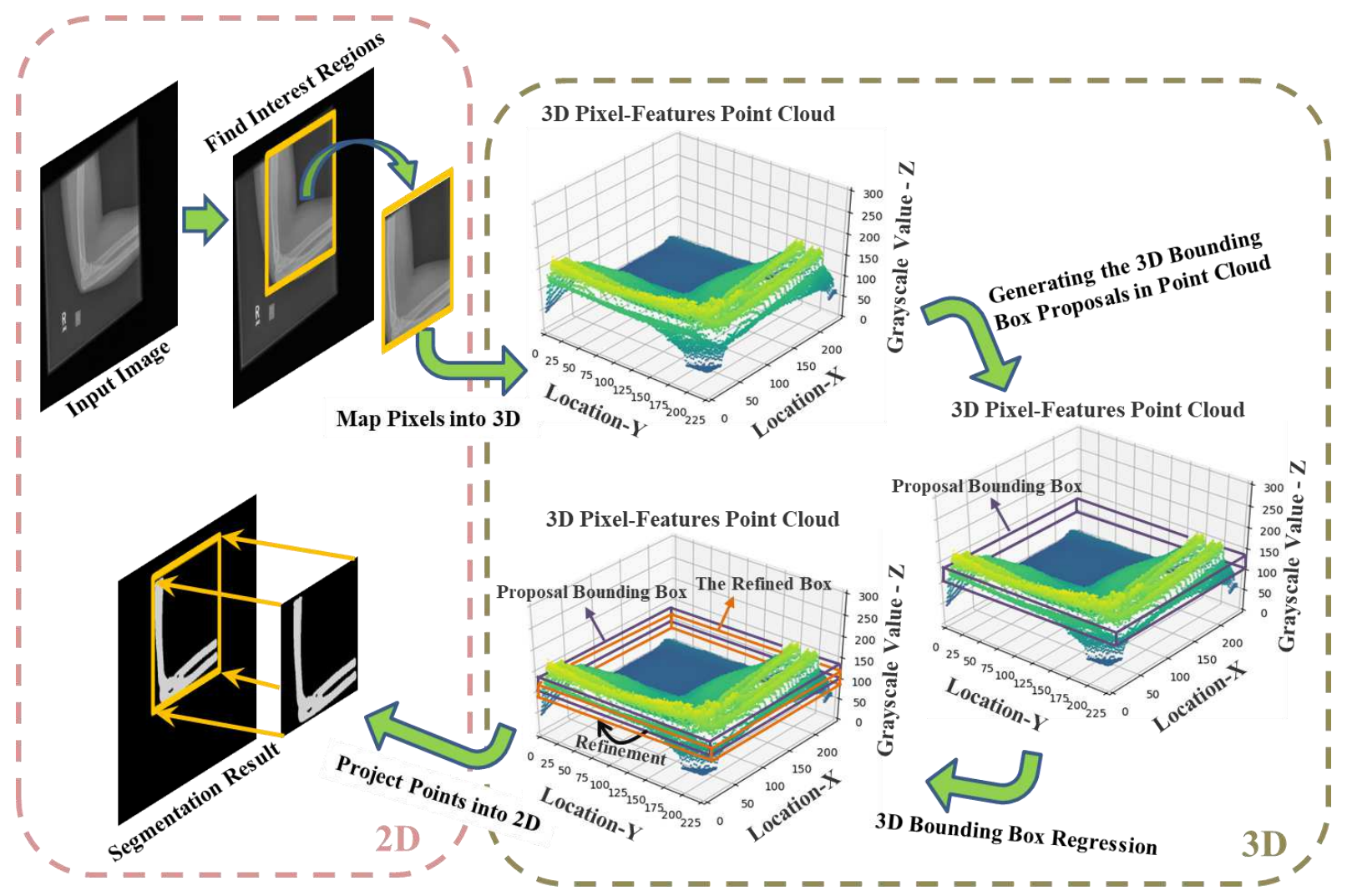

Fig.2 The pipeline of the proposed grayscale medical image segmentation method. 


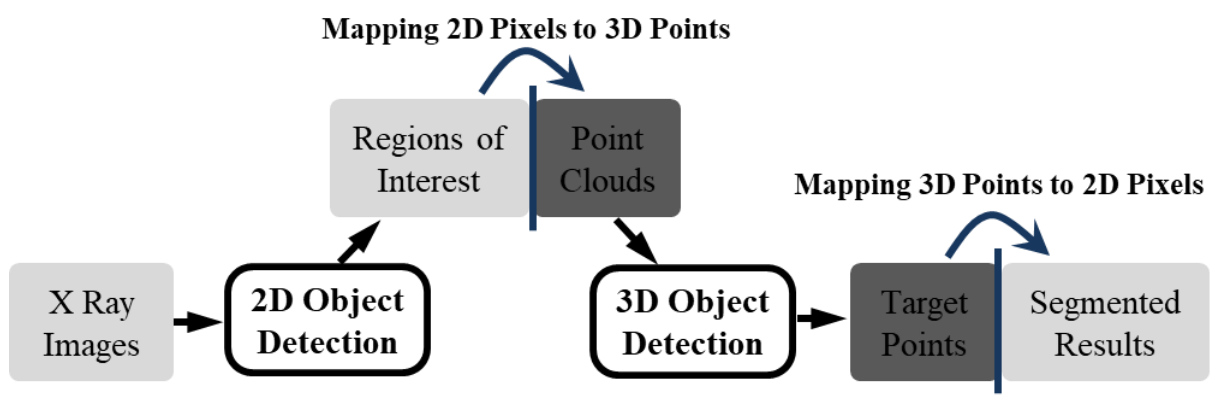

Fig.3 The implementation flow of the proposed method.

109 According to the proposed strategy and above pipeline, object detections play the central roles at

110 each block of our method. Many researches about 2D\&3D object detection has raised ever and

111 they could perform well especially those with deep learning.

112 The current mainstream 2D object detection methods based on deep learning could be generally 113 classified into two-stage and one-stage methods. [25] With two-stage methods, proposal bounding

114 boxes are generated firstly and the further refinement of proposals and confidences is obtained in

115 the second stage. [26] While using the one-stage methods [27,28], the location and the 116 classification of object bounding boxes could be estimated directly without refinement which

117 means one-stage methods are usually faster than two-stage ones but have lower object detection 118 accuracy. [29]

119 The widespread application of 3D geometric data spurs the development of 3D object detection 120 and it could be categorized into monocular/stereo image-based, point cloud-based and multimodal 121 fusion-based methods in terms of the modality of input data. [30] Due to point clouds are the most 122 regular data which could be achieved with different sensors, enormous researches of point cloud123 based methods have raised. [31-33] Among these method, different data format like raw point 124 clouds or 3D voxel grids transformed from points could be feed into deep net architectures to find 125 targets with bounding boxes and their classes. [34] 
127 In a medical grayscale image, pixels of the segmentation object always just take up a part of the 128 entire image and there may exists noisy pixels with the same gray values in irrelevant regions.

129 Therefore, 2D object detection is adopted as the pre-processing procedure to identify the specially 130 interest regions with segmentation objects and reduce noisy pixels as shown in Fig.4.
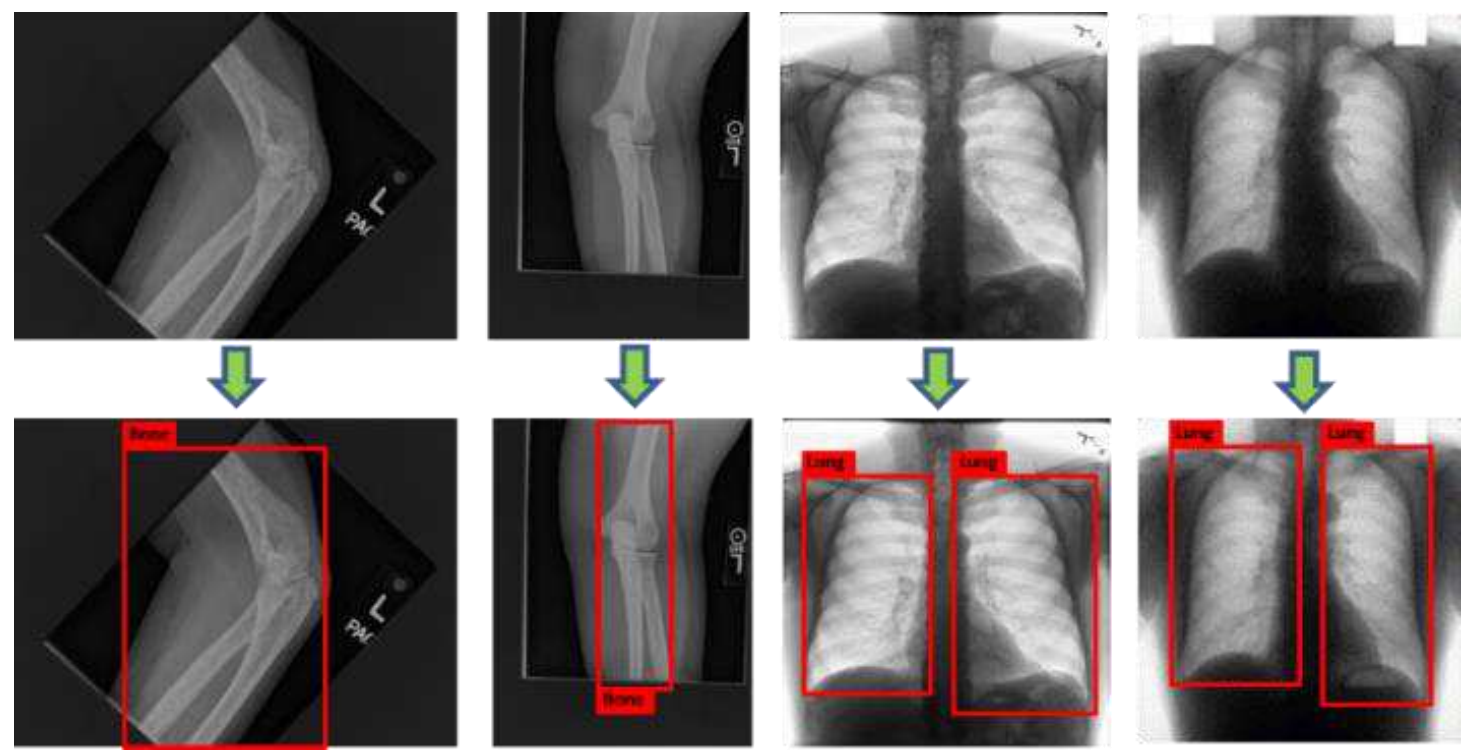

Fig.4 Achievement of interest regions in 2D images.

133 Compared with the accuracy, the proposed pre-processing procedure cares more about the

134 detection speed, so we adopt the one-stage method YOLOv3 [35,36] as the backbone network.

135 And considering the scarcity of labeled medical grayscale images, we apply the fine tuning - a

136 transfer learning method [29] to migrate most layers of the backbone model which was pretrained

137 on ImageNet, Pascal VOC (Pattern analysis, statistical modeling and computational learning visual

138 object classes) and MS COCO (Microsoft common objects in context) datasets. [37,38] As Fig.5.

139 shown, with fine tuning method, we could freeze N-M layers of pre-trained model and only train

140 the last M layers on local dataset. In order to retain the detection ability of pre-trained model as

141 much as possible, and ensure the stability of the loss change during the training process, the 
142 proposed image segmentation pre-processing method only unfreeze the last 3 layers of pre-trained 143 network for training.

144

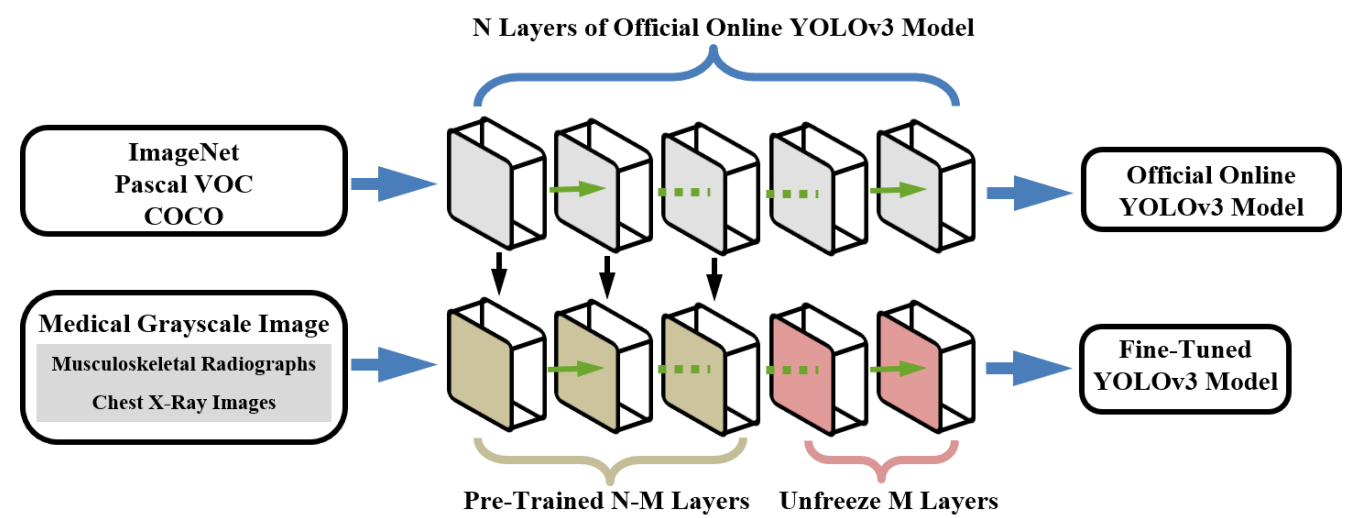

Fig.5 The proposed 2D object detection network with fine-tuning method.

\subsubsection{Generation of proposal bounding box in pixel-features point cloud}

The grayscale value of each pixel in interest regions represents their brightness. [39] Pixels compose the same tissues in particular image always share the grayscale value ranges and we could recognize them manually. All values range from 0 to 255 (Typically zero is taken to be black, and 255 is taken to be white). Darker pixels represent structures like soft tissues having less attenuation

to the beam, while light ones represent structures like bones having high attenuation. Due to the lack of detailed gray values of pixels displayed on 2D images, it is hard to determinate their specific grayscale value ranges.

Thus, we turn pixels in 2D interest regions into the 3D representations as Fig.6. shown. In Fig.6. the first two dimensions represent pixels locations and the third dimension represents their grayscale values. The 3D data could be considered as the pixel-features point cloud and it is distinct and intuitive to obtain points which represent pixels belong to the same tissues. This helps us translate the $2 \mathrm{D}$ image segmentation task into the $3 \mathrm{D}$ object detection with point cloud. We only need to determine locations and widths of 3D bounding boxes which contain the foreground points 
160 during the object detection. Then bottoms and tops of bounding boxes could represent the

161 segmentation required threshold values for 2D images.

162 Inspired by two-stage 2D object detection methods, we present a novel two-stage 3D object

163 detection method, which is operated on pixel-features point cloud. In the first stage of existing

164 popular two-stage 2D object detection method, the proposal bounding boxes with its classification

165 scores are generated with convolutional neural network and the refinements of those boxes are

166 obtained in the following stage after the Non-Maximum Suppression (NMS). While in our

167 proposed 3D object detection method, based on two-stage strategy, the proposal 3D bounding

168 boxes with the classification scores of points inside them are estimated firstly and these proposals

169 are refined with regression in second stage.
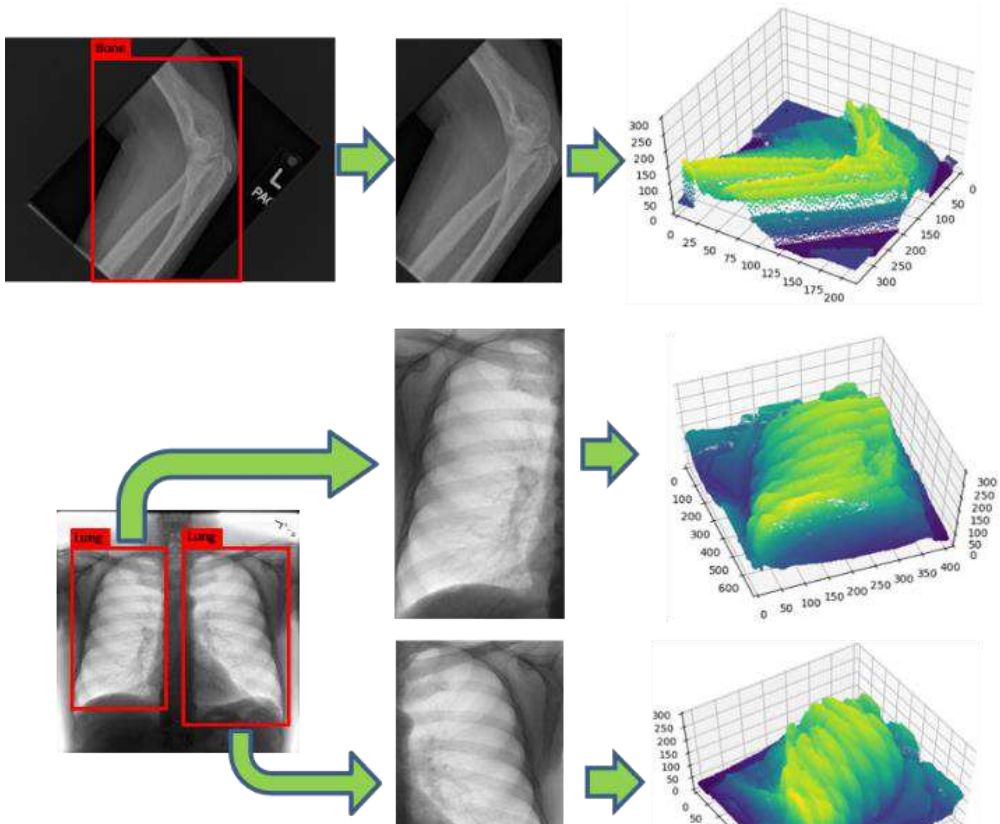

Fig.6 Turning pixels in interest regions into the pixel-features point cloud.

172 The generation of proposal bounding boxes in pixel-features point cloud has three modules. As

173 shown in Fig.7, These modules include localization of anchor boxes, classification of points inside 
174 boxes utilizing PointNet [34] as backbone network and Non-Maximum Suppression with 3D

175 Intersection-over-Union (IoU).

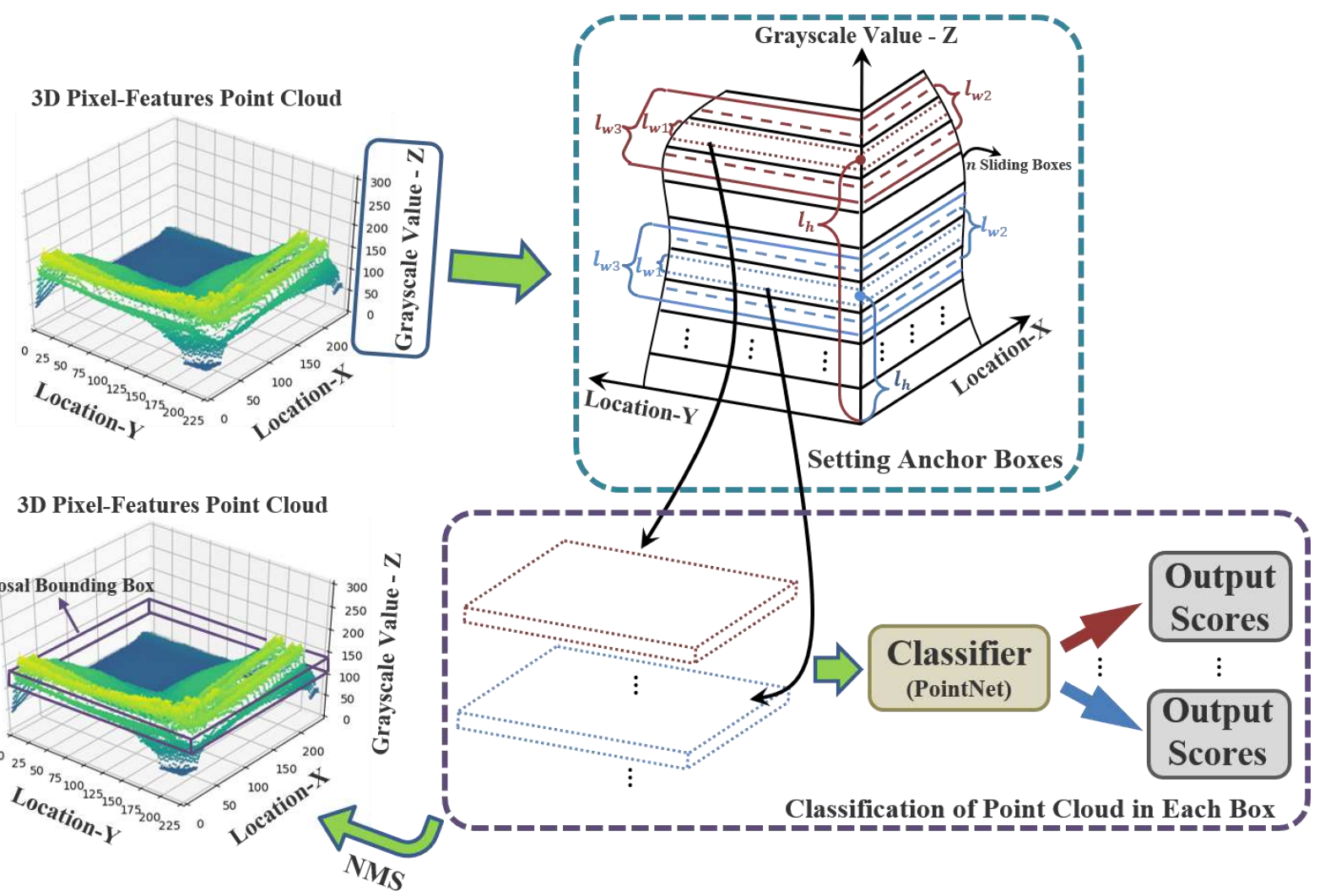

Fig.7 The generation of proposal bounding boxes in pixel-features point cloud.

179 Proposal bounding boxes generation takes the $l_{x} \times l_{y} \times 255$ point cloud representation as input 180 where $l_{x}$ and $l_{y}$ respectively indicate the length and width of $2 \mathrm{D}$ interest region. In order to avoid 181 high overlap rate of predict boxes and the low search efficiency using selective search as Region 182 Convolutional Neural Network (RCNN) method, inspired by the Region Proposal Networks 183 (RPN) in Faster RCNN, we apply the anchor boxes method for electing predict boxes.

184 To generate proposals, we slide a small network over the input by a shared 3D convolutional layer 185 referred to RPN and Single Shot MultiBox Detector (SSD) method as Fig.7. shown. At each 
186 sliding-box location, we could predict multiple proposals simultaneously, and we denote the

187 maximum number of possible proposals as $k$. These proposals are parameterized relative to $k$ 3D

188 anchor boxes. Each anchor is centered at its corresponding sliding box and is associated with a

189 scale. Each anchor is defined with coordinates $\left(l_{h}, l_{w}\right)$ where $l_{h}$ and $l_{w}$ represent its location and

190 scale. We apply 3 scales by default, deciding $k=3$ anchors at each sliding box and $n \times k$ anchors

191 in total.

\subsubsection{Classification of point cloud}

193 Anchor boxes with different scales share the same box-length $l_{x}$ and box-width $l_{y}$, and they are

194 distinguished by their center locations and box-heights. In order to determine the proposal

195 bounding box from numerous anchor boxes, we utilize the PointNet as our backbone network and

196 apply the fine-tuning method for training our classification module.

197 The classification network in Fig.7. indicates that raw point clouds are directly taken as the input

198 and each point is processed independently at the initial stage. Due to point clouds could be easily

199 applied rigid or affine transformations, input points are sorted into a canonical order with the first

200 affine transformation by a mini-net (T-net) and moreover, after points features extraction with

201 multi-layer perceptron (mlp), features from different points could also be aligned using another

202 alignment network by feature transformation matrix. Then, the max pooling layer aggregates all

203 points features extracted from the second mlp and outputs the global features. The final fully

204 connected layers set the global feature as input and outputs $k$ scores for all the $k$ candidate classes. 


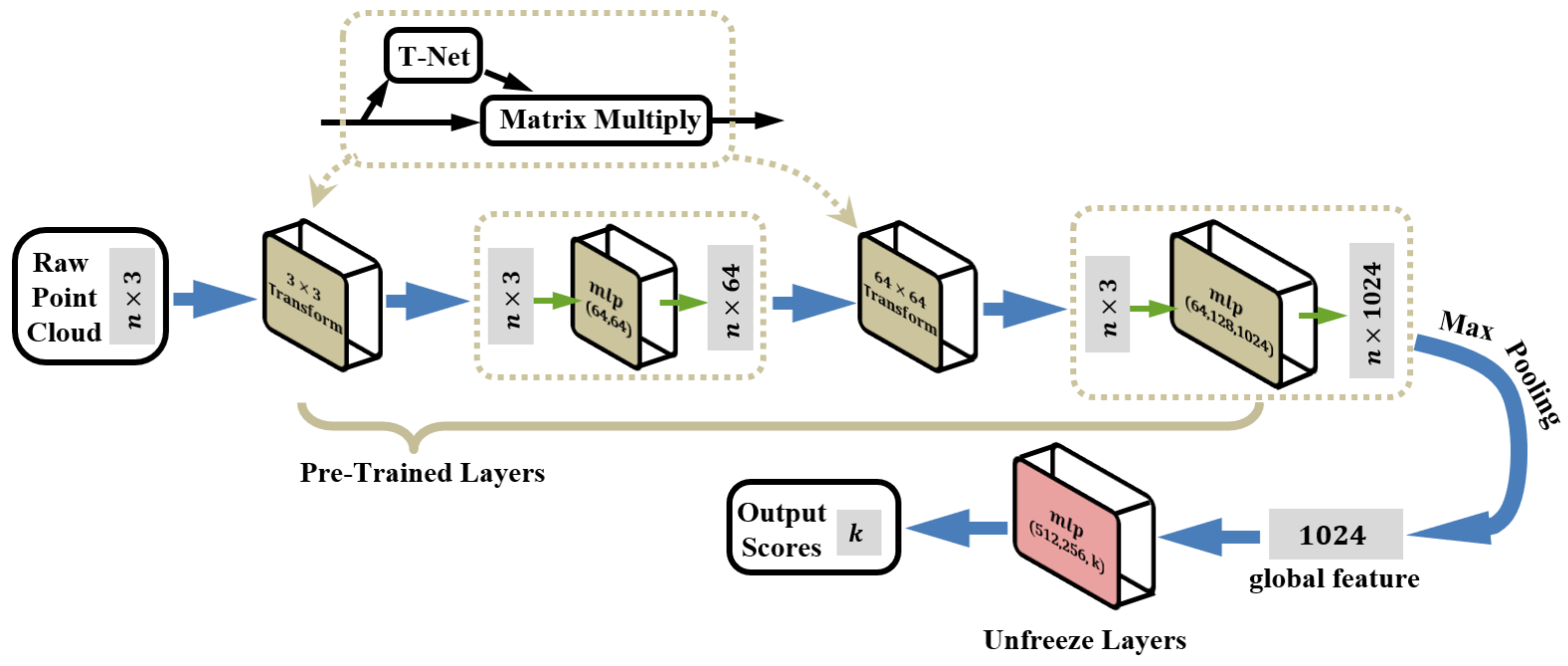

Fig.8 The proposed point cloud classification network with fine-tuning method.

207 It should be noted that models-based point clouds datasets which mapped from grayscale medical

208 images is scarce, thus we apply the fine-tuning method again. With the migration of PointNet

209 model pretrained on ModelNet40 [40], we freeze most layers of the network except the final fully

210 connected layers as shown in Fig.8.

212 After the above module, the classification results of point cloud in each anchor box could be 213 achieved with scores. But as many 2D object detection method, there exists some repeated 214 proposals of one object. They belong to the same candidate class and overlap with the local 215 highest-score box. For reducing the redundancy, we adopt the non-maximum NMS on these 216 proposals with 3D intersection over union (3D IoU). Different from the IoU computation for 2D 217 based on the relationships of areas between box $A$ and $B$ [41], like Fig.9. shows, volumes of two 218 boxes are applied for 3D IoU calculation [42] which could be formulated as:

$$
3 \mathrm{D} \operatorname{IoU}(A, B)=\frac{A_{v} \cap B_{v}}{A_{v} \cup B_{v}}=\frac{A_{v} \cap B_{v}}{\left|A_{v}\right|+\left|B_{v}\right|-A_{v} \cap B_{v}}
$$


221 only one box for each candidate class which could be considered as the proposal bounding box.

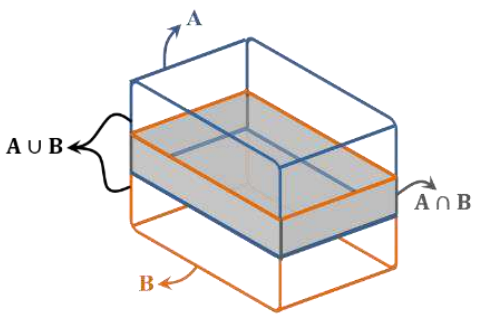

Fig.9 IoU computation for 3D. The intersection volume is highlighted in gray.

\section{4}

225

226

227

228

\subsubsection{Refinement of proposal bounding box}

Even though high classification scores of the proposal bounding boxes, the location and scale errors between them and ground truth exist. We train and implement a class-specific bounding box linear regression model to reduce errors and improve detection performance.

On the assumption that we achieve one proposal bounding box $P^{i}$ and its nearby ground-truth box $G^{i}$ as shown in Fig.10, where $P^{i}=\left(P_{l_{h}}^{i}, P_{l_{w}}^{i}\right)$ specifies height $l_{h}$ of the center of proposal bounding box together with its width $l_{w}$. Meanwhile, the ground-truth bounding box $G^{i}$ is specified in the same way: $G^{i}=\left(G_{l_{h}}^{i}, G_{l_{w}}^{i}\right)$. The goal of the bounding box regressor is to learn a transformation which could map each proposal bounding box $P$ to the ground-truth box $G$.

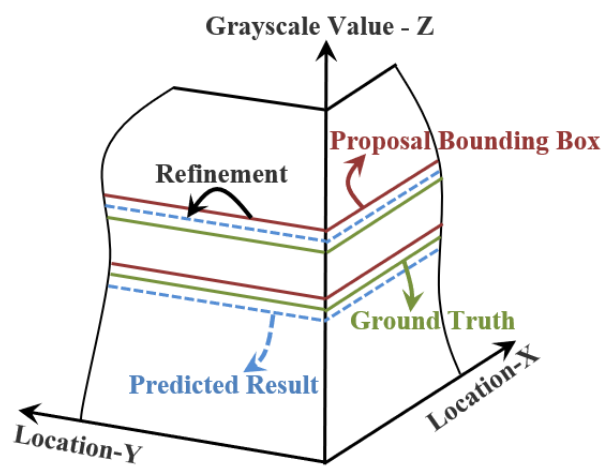

Fig.10 Refinement of proposal bounding box. 
235 The transformation could be parameterized in terms of two functions $d_{l_{h}}(P)$ and $d_{l_{w}}(P)$. The first

236 function specifies the translation of bounding box $P$ 's center which is scale-invariant, while the

237 second specifies the log-space translation of its width. By applying the transformation as following

238 equations, an input proposal bounding box $P$ could be transformed into a predicted ground-truth

239 box $\hat{G}$.

$$
\widehat{G}_{l_{h}}=P_{l_{w}} \times d_{l_{h}}(P)+P_{l_{h}}
$$

$$
\widehat{G}_{l_{w}}=P_{l_{w}} \times \exp \left(d_{l_{w}}(P)\right)
$$

242 Inspired by the 2D object detection, the bounding box regression of our method is performed on

243 global features which is max pooled from PointNet model. Above two functions $d_{l_{h}}(P)$ and

$244 d_{l_{w}}(P)$ could be modeled as linear functions of the global features of proposal bounding box $P$,

245 denoted as $f_{m p}(P)$. Therefore, we have $d_{*}(P)=\mathrm{T}_{*} \times f_{m p}(P)$, where $*$ represents $l_{h}$ or $l_{w}$, and $\mathrm{T}_{*}$

246 is a vector composed of learnable model parameters.

247 The transformation targets $t_{*}$ between proposal bounding box $P$ and the real ground-truth box $G$ 248 could be defined as:

$$
t_{l_{h}}=\frac{G_{l_{h}}-P_{l_{h}}}{P_{l_{w}}}
$$

$$
t_{l_{w}}=\log \left(\frac{G_{l_{w}}}{P_{l_{w}}}\right)
$$

251 Thus, after setting the loss function and by optimizing the regularized least squares objective as 252 following, we could learn $\mathrm{T}_{*}$ and achieve the transformation to refine the proposal bounding box.

$$
\text { Loss }=\sum_{i}^{N}\left(t_{*}{ }^{i}-\widehat{\mathrm{T}}_{*} \times f_{m p}\left(P^{i}\right)\right)^{2}
$$

$$
\mathrm{T}_{*}=\operatorname{argmin}_{\widehat{\mathrm{T}}_{*}} \operatorname{Loss}+\lambda\left\|\widehat{\mathrm{T}}_{*}\right\|^{2}
$$


256 The proposed grayscale medical image segmentation method follows a three-stage training 257 strategy. First, we obtain interest regions from raw grayscale images with fine-tuning YOLOv3 258 model. Second, by training the pixel-features point cloud classification model based on PointNet,

259 proposal 3D bounding boxes could be achieved from the point cloud representations of pixels in 260 interest regions. By training the linear regressor, proposal bounding boxes are refined with location 261 and scale transformation. Three independent modules including regions extractor, point cloud 262 classifier and bounding box regressor in three stages compose our method.

\subsection{Performance assessment}

264 In this study, we evaluate the segmentation performance by following four metrics: Dice similarity 265 coefficient (DSC) scores [6], intersection over union (IoU), False negative (FN) and False positive 266 (FP) [7]. Ranges of DSC and IoU are between 0 and 1, higher values of them and lower values of 267 FN and FP indicate the higher accuracy. The calculation formula of DSC is defined as:

$$
\mathrm{DSC}=\frac{2|T \cap G|}{|T|+|G|}
$$

269 where $T$ is the detected region and $G$ is the ground truth region.

\section{Results}

271 We conduct experiments by the proposed grayscale image segmentation method on above

272 mentioned datasets including musculoskeletal radiographs dataset and chest radiographs dataset.

273 Moreover, our prepared phalanx and forearm X ray images obtained with the portable X ray 274 machine as Fig.11. shown are also adopted for model training and validation. 


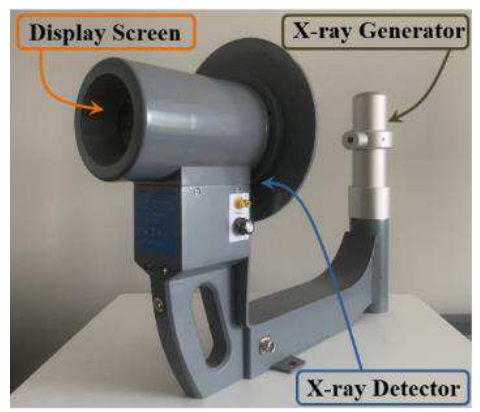

Fig.11 The portable X ray machine applied in experiments.

277 Our model is implemented with Pytorch [43] and its entire training process is performed on a 278 computer with Windows 10 operating system, Intel Core i7 processor with $3.0 \mathrm{GHz}, 64 \mathrm{~GB}$ of 279 RAM and a single NVIDIA GPU (Quadro RTX 4000). The 2D object detection model is trained 280 with 50 epochs for achieving interesting regions and it takes $1.75 \mathrm{~h}$, while the training of the 3D 281 object detection model for generating proposal bounding boxes spends $2.5 \mathrm{~h}$ on 200 epochs.

282 After training process, by applying the proposed method with the given grayscale medical images 283 input and following the method pipeline as Fig.2. shown, regions of target issues could be 284 segmented. Each block in Fig.12. presents several examples of segmentation performance from 285 different kinds of datasets, as well as processing results after each stage, where white represents 286 true positive pixels and black is for true negatives pixels. Moreover, according to evaluation 287 criteria, Table 1 shows four metrics including IoU, DSC, FN and FP to assess the segmentation 288 performance of images in different datasets.

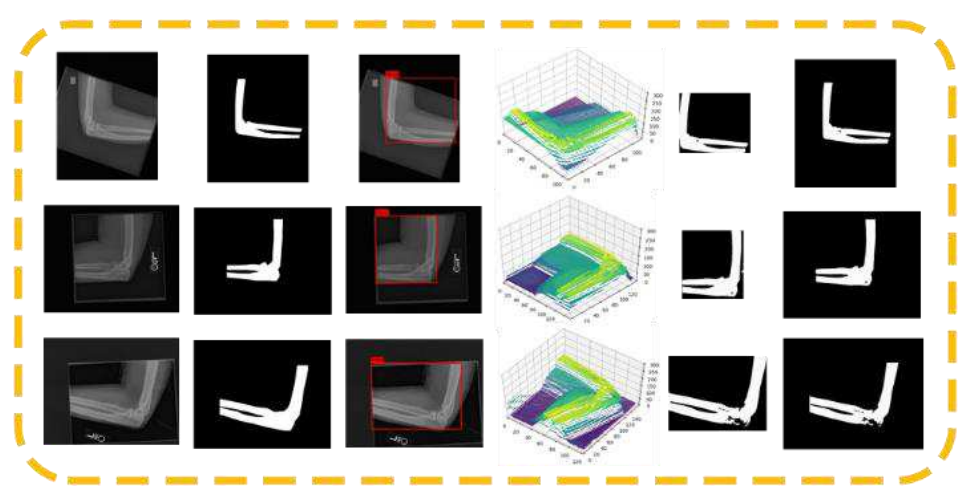

(a) Examples of segmentation performance in musculoskeletal radiographs dataset. 


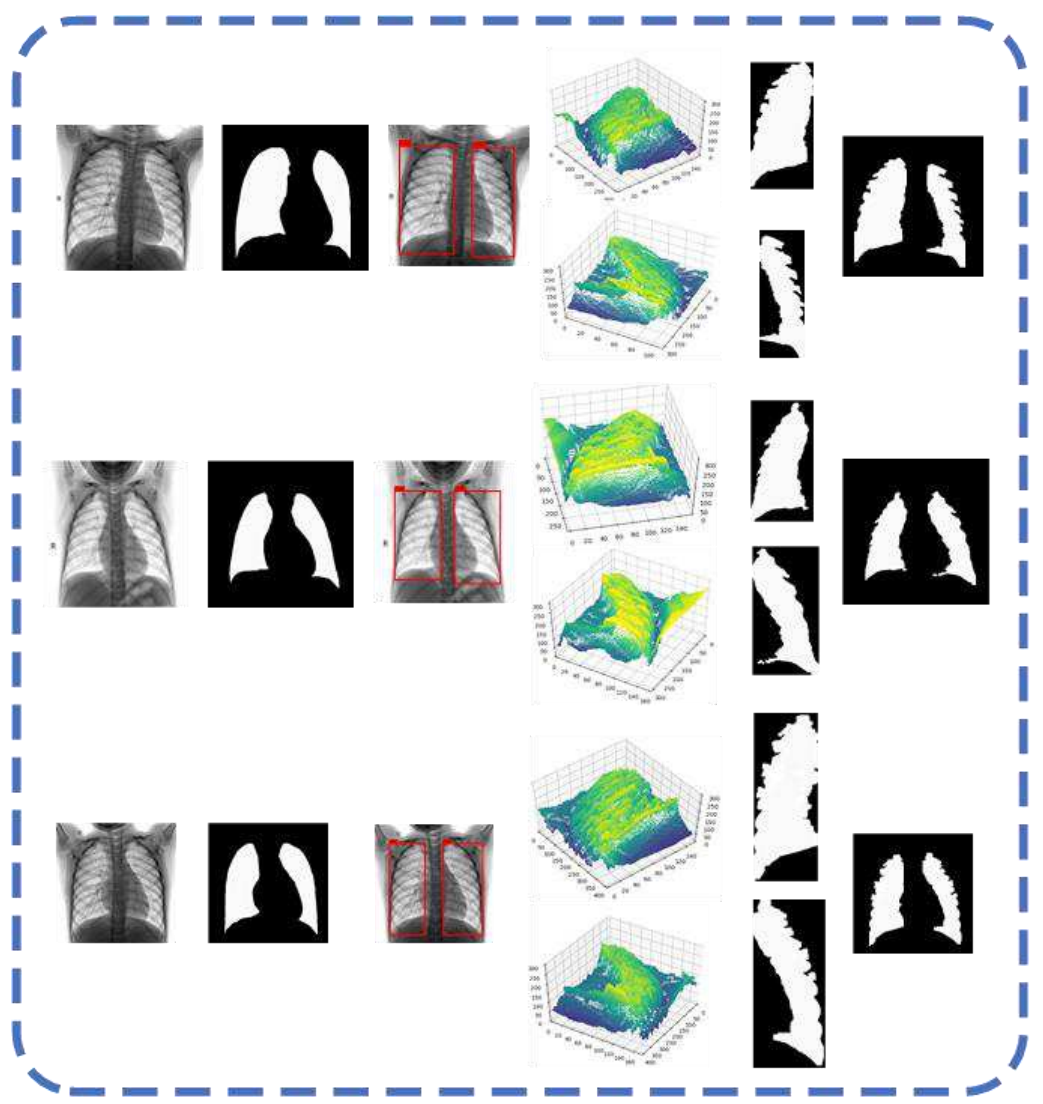

(b) Examples of segmentation performance in chest radiographs dataset.

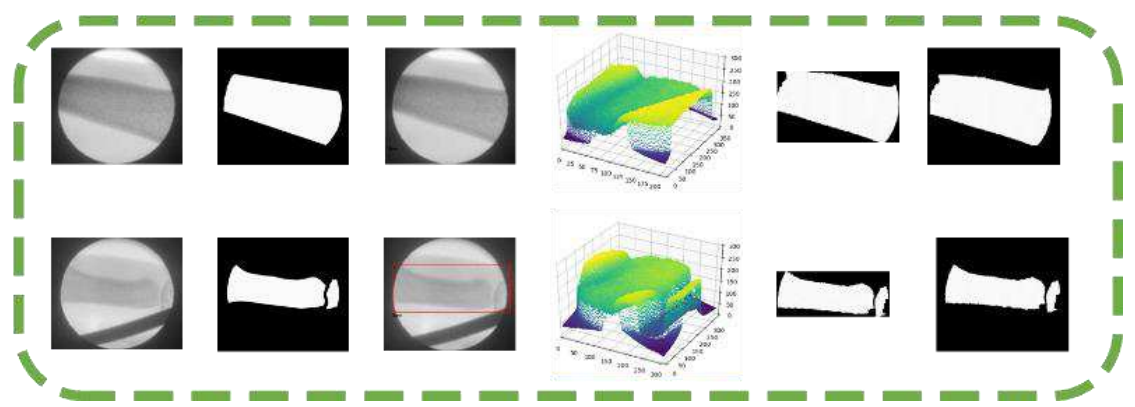

(c) Examples of segmentation performance in $\mathrm{X}$ ray images with the portable $\mathrm{X}$ ray machine.

295 Fig.12 Segmentation results from different kinds of datasets. From the first to the last column are origin images, ground truth,

296 achievements of interest regions, representations of pixel-feature point cloud, local segmentation results, and segmentation results in original image size, respectively.

Table 1 The values of evaluative metrics from experiments in different datasets.

\begin{tabular}{lllll}
\hline Datasets & IoU & DSC & FN & FP \\
\hline Musculoskeletal radiographs & 0.92 & 0.96 & 0.05 & 0.02 \\
Chest radiographs & 0.88 & 0.93 & 0.11 & 0.15 \\
Images from X ray machine & 0.94 & 0.94 & 0.06 & 0.08 \\
\hline
\end{tabular}


Table 2 Comparison between segmentation performance (IoU) of the proposed approach with other methods.

\begin{tabular}{lcccccc}
\hline Datasets & Proposed & FCN & UNet & UNet++ & Res-UNet & Attention Unet \\
\hline Musculoskeletal radiographs & 0.92 & 0.82 & 0.85 & 0.84 & 0.91 & 0.90 \\
Chest radiographs & 0.88 & 0.76 & 0.81 & 0.83 & 0.88 & 0.86 \\
Images from X ray machine & 0.94 & 0.72 & 0.82 & 0.87 & 0.85 & 0.91 \\
\hline
\end{tabular}
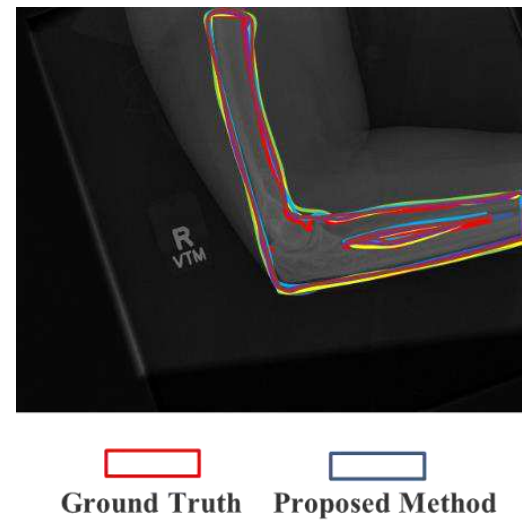
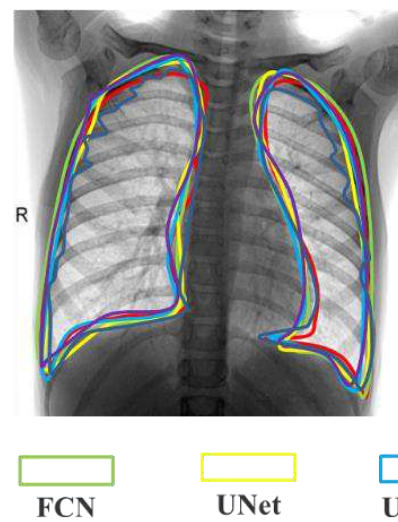

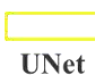

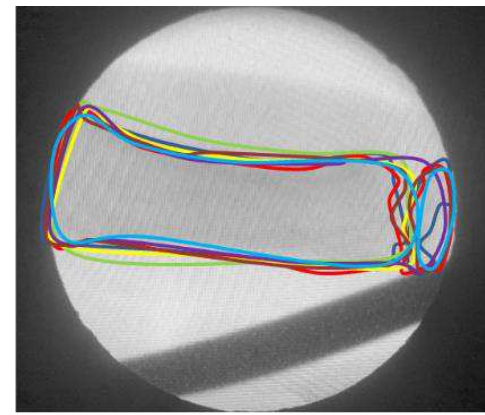

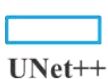

Res-UNet Attention UNet

Fig.13 Performance comparison of grayscale medical image segmentation with different methods.

302 As shown in Fig.12. and Table 1, we could obtain high IoU and DSC scores with satisficed

303 segmentation results on different datasets. This indicates that based on the proposed method, 2D

304 interest regions and 3D bounding boxes containing target pixel-features point cloud during the

305 processing could be successfully achieved.

\section{Discussion}

307 In this section, we compare the image segmentation performance of the proposed method with

308 multiple famous and clinically performed well models. As well known, CNN based models are

309 among the most successful and widely used for medical image processing. Besides the milestone

310 FCN model, UNet built on top of the fully convolutional networks with a U-shaped architecture

311 to capture context information, and based on it, Res-UNet [44] improved the segmentation results

312 using residual blocks as the building block and UNet++ [45] enhanced segmentation quality of

313 varying-size objects. Also, Attentiom UNet [46] achieved the better performance with the attention

314 gate. We train these models in the same dataset as our proposed method and Table 2 presents the 
315 comparison results. Meanwhile, Fig.13. shows results by visualization. It indicates that compared

316 with other models, our proposed approach improves the segmentation performance and it obtains

317 the highest IoU scores of $0.92,0.88$ and 0.94 with three datasets respectively. In our approach, 2D

318 and 3D object detection models could be both trained with transfer learning method which makes

319 it possible to achieve a quite accurate image segmentation model with small training datasets.

320 While other sematic segmentation methods may be sensitive to the scale of datasets because the

321 pre-trained model could only help simplify the downsample training procedure, and the training

322 of upsample still requires a number of datasets. This indicates that it is impossible to adapt them

323 for every application task well because training data is scare especially in medical image field.

324 Moreover, in grayscale images, grayscale values of pixels are important features to distinguish

325 different objects, and the intuitive logic of grayscale image segmentation could be considered as

326 the collection of pixels with similar grayscale values. So, the proposed image segmentation model

327 which obtains the purpose ranges of grayscale values with 3D object detection have better

328 explicability and segmentation effect.

329 Under different medical imaging devices and environment in clinical, ranges of grayscale values

330 of pixels which compose the same segmentation target in different medical images are always

331 different. But our proposed method could settle this and we could obtain thresholding values (top

332 and bottom of 3D bounding boxes) by mapping pixels in 2D images into 3D point clouds and

333 adopting 3D object detection with features of pixels.

\section{Conclusions}

335 In this paper, we present a new method for grayscale medical image segmentation only with two

336 object detection models. The method applies 2D object detection model for location identification

337 of segmentation objects. It could crop the origin images and increase the efficiency of further 
338 detailed segmentation. Pixels in interest regions are mapped as point cloud according to their

339 positions and grayscale values. Using 3D object detection methods, we achieve bounding boxes

340 which contain target pixels-feature points. After projecting these points to $2 \mathrm{D}$ images, they could

341 composite the segmentation results. The effectiveness of the proposed image segmentation method

342 is proven by several experiments in different image datasets and the comparison with other famous

343 approaches and it indicates the proposed method could perform better in grayscale image

344 segmentation tasks. In further research, we will concentrate on multi-oriented objects detection

345 technologies for more fine segmentation results.

\section{Abbreviations}

347 CNN(s): Convolutional neural network(s); FCN: Fully convolutional networks; MURA: 348 Musculoskeletal radiographs; LERA: Lower extremity radiographs; CheXpert : Chest radiography; 349 GT: Ground Truth; YOLO: You only look once; PASCAL VOC: Pattern analysis, statistical 350 modeling and computational learning visual object classes; MS COCO: Microsoft common objects 351 in context; NMS: Non-maximum suppression; IoU: Intersection-over-Union; RCNN: Region 352 convolutional neural network; RPN: Region proposal networks; SSD: Single shot multiBox 353 detector; mlp: multi-layer perceptron; DSC: Dice similarity coefficient; FN: False negative; FP: 354 False positive.

\section{Declarations}

\section{Ethics approval}

357 We declare that all of us obey the principles of the Declaration of Helsinki. In other words, all 358 experiments and methods in this paper are in accordance with these principles. The study was 359 approved by the Ethics Committee of the First people's Hospital of Yancheng.

\section{Consent to participate}

361 The fully anonymized phalanx and forearm X ray images were received by authors on 2 April, 3622021 and the requirement for informed consent was waived for this study because of the 363 anonymous nature of the data. 


\section{Consent for publication}

365 Not applicable for this paper

Availability of data and materials

367 Musculoskeletal radiographs and chest radiographs which support our research are available from 368 Stanford ML Group. But restrictions apply to the availability of these data, which were used under 369 license for the current study, and so are not publicly available. Data are however available from 370 the authors upon reasonable and with permission of Stanford ML Group. While phalanx and 371 forearm $\mathrm{X}$ ray images are available only upon request by emailing authors due to the ethical 372 restrictions on sharing these data which could contain potentially sensitive information of patients.

\section{Competing interests}

374 All authors declare that they have no interest conflicts or competing interests.

375 Founding

376 This work was supported by the project of Tongji University Sheng Feiyun College Student 377 Science and Technology Innovation Practice Found.

\section{Authors' contributions}

379 Qing Zhang conceived the research. Yunfei Ge and Yidong Shen analyzed the clinical and imaging 380 data. Yuantao Sun, Yunfei Ge, and Xijiong Wang designed the study. Yunfei Ge and Yidong Shen 381 performed the experiments and collected the results. Yunfei Ge and Yuantao Sun drafted the 382 manuscript. Qing Zhang reviewed the final manuscript. All authors read and approved the final 383 manuscript.

\section{Acknowledgements}

385 Not applicable.

\section{References}

1. Justine Wallyn, Anton Nicolas, Akram Salman, et al. Biomedical imaging: principles, technologies, clinical aspects, contrast agents, limitations and future trends in nanomedicines. Pharmaceutical Research. 2019; 36(6):78-108.

2. Yeo W K, Yap D F W, et al. Grayscale medical image compression using feedforward neural networks. 2011 IEEE International Conference on Computer Applications and Industrial Electronics (ICCAIE). 2011; 633-638.

3. Lei Tao, et al. Medical Image Segmentation Using Deep Learning: A Survey. arXiv. 2020; 13120.

4. Rathnayaka K, Sahama T, Schuetz MA, et al. Effects of CT image segmentation methods on the accuracy of long bone 3D reconstructions. Medical Engineering \& Physic. 2011; 33(2): 226-233. 
5. Shuo Wang, Zhou Mu, Liu Zaiyi, et al. Central focused convolutional neural networks: Developing a data-driven model for lung nodule segmentation. Medical Image Analysis. 2017; 40: 172-183.

6. Han Liu, Wang Lei, Nan Yandong, et al. SDFN: Segmentation-based deep fusion network for thoracic disease classification in chest X ray images. Computerized Medical Imaging and Graphics. 2019; 75: 66-73.

7. de Albuquerque VHC, Rodrigues D A, Ivo RF, et al. Fast fully automatic heart fat segmentation in computed tomography datasets. Computerized Medical Imaging and Graphics. 2020; 80: 101674.

8. Li Wen, et al. Automatic segmentation of liver tumor in CT images with deep convolutional neural networks. Journal of Computer and Communications. 2015; 3(11): 146.

9. Vivanti R, Ephrat A, Joskowicz L, et al. Automatic liver tumor segmentation in follow-up CT studies using convolutional neural networks. Proc. Patch-Based Methods in Medical Image Processing Workshop. 2015; 2: 2.

10. Saleha Masood, Sharif Muhammad, Masood Afifa, et al. A survey on medical image segmentation. Current Medical Imaging. 2015; 11(1): 3-14.

11. Khandare ST, Isalkar A D. A survey paper on image segmentation with thresholding. International Journal of Computer Science and Mobile Computing. 2014; 3(1): 441-446.

12. Sezgin M, Sankur B. Survey over image thresholding techniques and quantitative performance evaluation. Journal of Electronic Imaging. 2004; 13(1): 146-165.

13. Maolood I Y, Al-Salhi Y E A, Lu S. Thresholding for medical image segmentation for cancer using fuzzy entropy with level set algorithm. Open Medicine. 2018; 13(1): 374-383.

14. Duo Hao, Li Qiuming, Li Chengwei. Histogram-based image segmentation using variational mode decomposition and correlation coefficients. Signal, Image and Video Processing. 2017; 11(8): 14111418.

15. Long J, Shelhamer E, Darrell T. Fully convolutional networks for semantic segmentation. Proceedings of the IEEE Conference on Computer Vision and Pattern Recognition (CVPR). 2015; 3431-3440.

16. Ronneberger O, Fischer P, Brox T. U-net: Convolutional networks for biomedical image segmentation. International Conference on Medical Image Computing and Computer-Assisted Intervention (MICCAI). 2015; 234-241.

17. Chen LC, Papandreou G, Kokkinos I, et al. Deeplab: Semantic image segmentation with deep convolutional nets, atrous convolution, and fully connected crfs. IEEE Transactions on Pattern Analysis and Machine Intelligence. 2017; 40(4): 834-848.

18. Deng J, Dong W, Socher R, et al. Imagenet: A large-scale hierarchical image database. Proceedings of the IEEE Conference on Computer Vision and Pattern Recognition. 2009; 248-255.

19. Kalinin A A, Iglovikov V I, Rakhlin A, et al. Medical image segmentation using deep neural networks with pre-trained encoders. Deep Learning Applications. 2020; 39-52.

20. Pierre-Henri Conze, Brochard Sylvain, Burdin Val-E-Rie, et al. Healthy versus pathological learning transferability in shoulder muscle MRI segmentation using deep convolutional encoder-decoders. Computerized Medical Imaging and Graphics. 2020; 83: 101733.

21. Pranav Rajpurkar, Irvin Jeremy, Bagul Aarti, et al. Mura: Large dataset for abnormality detection in musculoskeletal radiographs. arXiv. 2017; 1712.06957.

22. LERA - lower extremity radiographs. https://aimi.stanford.edu/lera-lower-extremity-radiographs-2.

23. Irvin J, Rajpurkar P, Ko M, et al. Chexpert: A large chest radiograph dataset with uncertainty labels and expert comparison. Proceedings of the AAAI Conference on Artificial Intelligence. 2019; 33(01): 590-597.

24. Joseph-Paul Cohen, Morrison Paul, Dao Lan, et al. Covid-19 image data collection: Prospective predictions are the future. arXiv. 2020; 2006.11988.

25. Jiao L, Zhang F, Liu F, et al. A survey of deep learning-based object detection. IEEE Access. 2019; 7:128837-128868.

26. Ross Girshick, Donahue Jeff, Darrell Trevor, et al. Rich feature hierarchies for accurate object detection and semantic segmentation. Proceedings of the IEEE Conference on Computer Vision and Pattern Recognition. 2014; 580-587. 
27. Joseph Redmon, Divvala Santosh, Girshick Ross, et al. You only look once: Unified, real-time object detection. Proceedings of the IEEE Conference on Computer Vision and Pattern Recognition. 2016; 779-788.

28. Wei Liu, Anguelov Dragomir, Erhan Dumitru, et al. SSD: Single shot multibox detector. European Conference on Computer Vision. 2016; 21-37.

29. Shin HC, Roth H R, Gao M, et al. Deep convolutional neural networks for computer-aided detection: $\mathrm{CNN}$ architectures, dataset characteristics and transfer learning. IEEE Transactions on Medical Imaging. 2016; 35(5): 1285-1298.

30. Qian R, Lai X, Li X. 3D Object Detection for Autonomous Driving: A Survey. arXiv. 2021; 2106.10823.

31. Zhou Y, Tuzel O. Voxelnet: End-to-end learning for point cloud based 3d object detection. Proceedings of the IEEE conference on computer vision and pattern recognition. 2018: 4490-4499.

32. Chen Y, Liu S, Shen X, et al. Fast point r-cnn. Proceedings of the IEEE/CVF International Conference on Computer Vision. 2019: 9775-9784.

33. Shi S, Wang X, Li H P. 3d object proposal generation and detection from point cloud. Proceedings of the IEEE Conference on Computer Vision and Pattern Recognition, Long Beach, CA, USA. 2019: 1620.

34. Qi CR, Su H, Mo K, et al. Pointnet: Deep learning on point sets for 3d classification and segmentation. Proceedings of the IEEE Conference on Computer Vision and Pattern Recognition. 2017; 652-660.

35. Redmon J, Farhadi A. Yolov3: An incremental improvement. arXiv. 2018; 1804.02767.

36. Rasmus Rothe, Guillaumin Matthieu, Van Gool Luc. Non-maximum suppression for object detection by passing messages between windows. Asian Conference on Computer Vision. 2014; 290-306.

37. Everingham M, Van Gool L, Williams C K, et al. The pascal visual object classes (voc) challenge: A Retrospective. International Journal of Computer Vision. 2014; 111: 98-136.

38. Lin T Y, Maire M, Belongie S, et al. Microsoft COCO: Common Objects in Context. European Conference on Computer Vision. 2014; 740-755.

39. Tan L, Jiang J. Digital signal processing: fundamentals and applications. Academic Press; 2019.

40. Zhirong Wu, Song Shuran, Khosla Aditya, et al. 3d shapenets: A deep representation for volumetric shapes. Proceedings of the IEEE Conference on Computer Vision and Pattern Recognition. 2015; 19121920.

41. Hamid Rezatofighi, Tsoi Nathan, Gwak JunYoung, et al. Generalized intersection over union: A metric and a loss for bounding box regression. Proceedings of the IEEE Conference on Computer Vision and Pattern Recognition. 2019; 658-666.

42. Zhou D, Fang J, Song X, et al. Iou loss for $2 \mathrm{~d} / 3 \mathrm{~d}$ object detection. International Conference on 3D Vision (3DV). 2019; 85-94.

43. Adam Paszke, Gross Sam, Massa Francisco, et al. Pytorch: An imperative style, high-performance deep learning library. Advances in Neural Information Processing Systems. 2019; 32: 8026-8037

44. X. Xiao, S. Lian, Z. Luo and S. Li. Weighted Res-UNet for High-Quality Retina Vessel Segmentation. 2018 9th International Conference on Information Technology in Medicine and Education (ITME). 2018; 327-331.

45. Z. Zhou, M. M. R. Siddiquee, N. Tajbakhsh and J. Liang. UNet++: Redesigning Skip Connections to Exploit Multiscale Features in Image Segmentation. IEEE Transactions on Medical Imaging. 2020; 39(6): 1856-1867.

46. Ozan Oktay, Jo Schlemper, et al. Attention U-Net: Learning Where to Look for the Pancreas. arXiv. 2018; 1804.03999. 